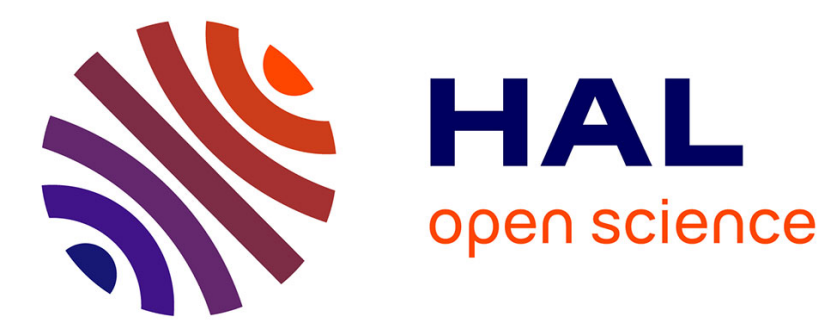

\title{
Scattering proton CT
}

Nils Krah, Catherine Therese T Quiñones, Jean-Michel Létang, Simon Rit

\section{To cite this version:}

Nils Krah, Catherine Therese T Quiñones, Jean-Michel Létang, Simon Rit. Scattering proton CT. Physics in Medicine and Biology, 2020, 65 (22), pp.225015. 10.1088/1361-6560/abbd18. hal02959263

\section{HAL Id: hal-02959263 https://hal.science/hal-02959263}

Submitted on 6 Oct 2020

HAL is a multi-disciplinary open access archive for the deposit and dissemination of scientific research documents, whether they are published or not. The documents may come from teaching and research institutions in France or abroad, or from public or private research centers.
L'archive ouverte pluridisciplinaire HAL, est destinée au dépôt et à la diffusion de documents scientifiques de niveau recherche, publiés ou non, émanant des établissements d'enseignement et de recherche français ou étrangers, des laboratoires publics ou privés. 


\title{
Scattering proton CT
}

\author{
N Krah ${ }^{1,2}$, CT Quiñones ${ }^{1}$, JM. Létang ${ }^{1}$, S Rit $^{1}$ \\ ${ }^{1}$ University of Lyon, CREATIS; CNRS UMR5220, Inserm U1044, INSA-Lyon, Université Lyon 1, Centre Léon Bérard, \\ France \\ ${ }^{2}$ University of Lyon, Université Claude Bernard Lyon 1, CNRS/IN2P3, IP2I Lyon, F-69622, Villeurbanne, France. \\ nils.krah@creatis.insa-lyon.fr
}

\begin{abstract}
Proton computed tomography $(\mathrm{CT})$ is an imaging modality investigated mainly in the context of proton therapy as a complement to x-ray CT. It uses protons with high enough energy to fully traverse the imaged object. Common prototype systems measure each proton's position and direction upstream and downstream of the object as well as the energy loss which can be converted into the water equivalent thickness. A reconstruction algorithm then produces a map of the relative stopping power in the object. As an alternative to energy-loss proton CT, it has been proposed to reconstruct a map of the object's scattering power based on the protons' angular dispersion which can be estimated from the measured directions. As in energy-loss proton $\mathrm{CT}$, reconstruction should best be performed considering the non-linear shape of proton trajectories due to multiple Coulomb scattering, but no algorithm to achieve this is so far available in the literature.

In this work, we propose a filtered backprojection algorithm with distance-driven binning to account for the protons' most likely path. Furthermore, we present a systematic study of scattering proton $\mathrm{CT}$ in terms of inherent noise and spatial resolution and study the artefacts which arise from the physics of multiple Coulomb scattering. Our analysis is partly based on analytical models and partly on Monte Carlo simulations.

Our results show that the proposed algorithm performs well in reconstructing relative scattering power maps, i.e. scattering power relative to that of water. Spatial resolution is improved by almost a factor of three compared to straight line projection and is comparable to energy-loss proton CT. Image noise, on the other hand, is inherently much higher. For example, in a water cylinder of $20 \mathrm{~cm}$ diameter, representative of a human head, noise in the central image pixel is about 40 times higher in scattering proton CT than in energy-loss proton CT. Relative scattering power in dense regions such as bone inserts is systematically underestimated by a few percent, depending on beam energy and phantom geometry.
\end{abstract}

This is a peer-reviewed, un-copyedited version of an article accepted for publication/published in Physics in Medicine and Biology. IOP Publishing Ltd is not responsible for any errors or omissions in this version of the manuscript or any version derived from it. The Version of Record is available online at https://doi.org/10.1088/1361-6560/abbd18. 


\section{Introduction}

The use of charged particles in medical imaging has been proposed long ago by Cormack (1963) and Koehler (1968) and later by Cormack et al. (1976), Hanson et al. (1981), and Hanson et al. (1982). While initially discarded in disfavour of x-ray computed tomography (CT), proton $\mathrm{CT}$ has seen renewed interest more recently in the context of proton therapy. We refer to Schulte et al. (2012), Poludniowski et al. (2015), and Johnson (2018) for recent reviews. Proton CT in its common implementation directly provides a relative stopping power (RSP) image of the patient - a quantity needed by treatment planning systems. This is expected to be an advantage over the currently used x-ray planning CT images which need to be translated into relative stopping power by means of an approximate conversion.

In this work, we focus on so-called "single tracking" or "list-mode" proton CT scanners, i.e. systems which record the kinematic properties of individual protons. Typically, these systems measure each proton's position and direction upstream and downstream of the imaged object, as well as some quantity indicative of the energy lost by the proton while traversing the object. Independently of the detector hardware employed to achieve this, the reconstructed quantity is the RSP. We will henceforth refer to this modality as energy-loss proton CT.

The reconstruction problem in proton $\mathrm{CT}$ is in principal similar to the one in x-ray $\mathrm{CT}$ in that it involves line integrals across the imaged object. A peculiar trait of proton $\mathrm{CT}$ is that proton trajectories are stochastic and not straight because protons undergo multiple Coulomb scattering (MCS). This is commonly accounted for by estimating the most likely path (MLP) for each proton and performing line integrals along the resulting curvilinear lines (Williams, 2004; Schulte et al., 2008; Erdelyi, 2009; Collins-Fekete et al., 2016; Krah et al., 2019).

In addition to energy-loss proton $\mathrm{CT}$, other contrast mechanisms have been proposed, which exploit different types of interaction of protons with a medium. In particular, these are attenuation and scattering proton CT. The former measures the reduction in proton fluence after an object due to inelastic nuclear interactions and reconstructs a map of the nuclear attenuation coefficient (Quiñones et al., 2016). The latter estimates the angular dispersion of protons due to MCS in the object and reconstructs a parameter describing MCS, e.g. radiation length (Bopp et al., 2013; Bopp et al., 2015; Taylor et al., 2016). An appealing practical aspect of scattering proton $\mathrm{CT}$ is that the imaging system is simpler than in energy-loss proton $\mathrm{CT}$ : it only requires tracking devices (to measure a proton's direction upstream and downstream of the object), but no detector to measure a proton's residual energy. It has also been observed that scattering proton CT might provide higher contrast images than energy-loss proton CT (Plautz et al., 2014).

At the same time, there is currently very limited literature available about reconstruction algorithms for scattering proton CT which use the MLP. Quiñones et al. (2016) present a filtered backprojection reconstruction method for attenuation proton CT which incorporates the MLP via distance driven binning, as initially proposed by Rit et al. (2013). Bopp et al. (2015) reconstruct scattering proton CT images via iterative reconstruction yet without considering the MLP. Taylor et al. (2016) have used a backprojection-then-filtration algorithm (Poludniowski et al., 2014), but provide relatively few details. In this work, we present a distance-driven binning reconstruction for scattering proton CT which takes into account the MLP.

Furthermore, we analyse systematically the potential performance of scattering proton CT and compare it with the more common contrast mechanism, i.e. energy-loss proton $\mathrm{CT}$, as well as attenuation pCT. Such a study is, to our knowledge, also missing in the literature so far. In particular, we investigate artefacts in scattering proton $\mathrm{CT}$ and their origin. We analyse the intrinsic lower limits in terms of image noise and finally quantify the typical signal-to-noise ratio, contrast-to-noise ratio, and spatial resolution achievable in scattering proton CT. We perform our study partly based on analytical models and partly based on Monte Carlo simulations. 


\section{Materials and Methods}

\subsection{Rationale of scattering proton CT}

The stochastic interaction of protons with the Coulomb field of nuclei in a medium results in multiple deviations from the protons' original direction. This phenomenon is referred to as multiple Coulomb scattering (MCS) (Gottschalk, 2010). Let the proton beam be aligned with the $z$-axis, then the quantities of interest are the angular deviations $\theta_{x}$ and $\theta_{y}$ as well as the lateral deviation $t_{x}$ and $t_{y}$ in the two planes perpendicular to the beam axis, respectively. Henceforth, we will drop the index $x$ or $y$ for simplicity and tacitly assume that angles are projected onto one of the two transversal planes.

We denote with $A \equiv\langle\theta\rangle^{2}$ the variance of the angular distribution in some depth $z$ and define the scattering power as the rate of change of the angular variance with depth,

$$
T(z)=\frac{\mathrm{d} A(z)}{\mathrm{d} z} \quad \Leftrightarrow A(z)=\int_{0}^{z} T\left(z^{\prime}\right) \mathrm{d} z^{\prime}+A_{0} .
$$

This definition is in analogy to the (energy) stopping power $\mathrm{d} E(z) / \mathrm{d} z$. We will henceforth consider $A_{0}=0$, without loss of generality. The rational behind scattering proton CT is to measure $A$ or a related quantity and reconstruct the scattering power $T$ of the traversed tissue. It is important to note, however, that $T$ is not a purely material specific local quantity, but also depends on the proton energy $E(z)$ at depth $z$ which in turn depends on the initial energy $E_{\mathrm{in}}$ and the type and amount of tissues upstream of $z$. In this work, we explore if and how a scattering power map could be reconstructed using analytical reconstruction methods.

\subsection{Relative scattering power}

In this section, we present how analytical methods used in energy-loss pCT could be applied to scattering $\mathrm{pCT}$. To start off, we recall that the proton stopping power $\mathrm{d} E / \mathrm{d} z$ depends on the proton energy (Paganetti, 2012). In fact, this reciprocal dependency is what leads to the sharp Bragg peak in depth dose profiles and what gives proton therapy its characteristic dosimetric properties. However, the ratio of stopping powers in human tissue relative to that of water is almost independent of energy (Arbor et al., 2015). It can therefore be considered a local tissue property which upon integration along a projection line gives the water equivalent path length making energy-loss pCT a well-defined tomographic problem. We will derive the tomographic problem of scattering pCT based on the concept of relative scattering power.

In this section, we consider as spatial variable the depth $z$ along a certain line of projection under a certain projection angle, starting at $z=0$ where the protons enter the object. We will later generalize the variable $z$ to account for the most likely path, but wish to keep notation as simple as possible here.

We use the radiation length $X_{0}$ to parametrize the degree of MCS in a medium, so that the scattering power $\mathrm{d} A / \mathrm{d} z=T\left(X_{0}(z), E(z)\right)$ depends on the proton energy $E(z)$ at $z$ and on the radiation length $X_{0}(z)$ (Gottschalk, 2010). The quantity $X_{0}(z)$ is purely local, i.e. it only depends on the local tissue properties, while the term $E(z)$ depends on the material traversed by a proton before reaching $z$ and therefore on the projection angle.

We observe that

$$
\begin{aligned}
& f: z \mapsto A(z) \text { and } \\
& g: z \mapsto E(z)
\end{aligned}
$$

are always monotonous functions because the proton energy always decreases and the angular dispersion always increases. Therefore, the mapping

$$
h \equiv g \circ f^{-1}
$$


is always bijective. In more practical terms, $E=h(A)$ is the energy of an initially co-linear proton beam in a medium at a depth where its angular dispersion is $A$. We can therefore define the scattering power as a function of angular variance,

$$
\tau\left(X_{0}(z), A(z)\right) \equiv T\left(X_{0}(z), h(A(z))\right) .
$$

We note that the function $h$ is not unique because $A(z)$ and $E(z)$ depend on the composition of the medium and the line of projection along which $z$ is taken, unless the medium is homogeneous. With $h_{w}$ the mapping in water, we define the scattering power in water as a function of $A$,

$$
\tau_{w}(A) \equiv T\left(X_{0, w}, h_{w}(A(z))\right)
$$

and the relative scattering power

$$
\delta\left(X_{0}(z), A(z)\right)=\frac{\tau\left(X_{0}(z), A(z)\right)}{\tau_{w}(A)} .
$$

Combining equations 1 and 7 , we obtain the integral expression

$$
G\left(A_{\text {out }}\right)=\int_{0}^{A_{\text {out }}} \frac{1}{\tau_{w}(A)} \mathrm{d} A=\int_{0}^{z_{\text {out }}} \delta\left(X_{0}(z), A(z)\right) \mathrm{d} z .
$$

The left-hand side can be interpreted as the water equivalent scattering path length $G$, i.e. the thickness of a block of water needed to generate the angular dispersion $A_{\text {out }}$. This is in analogy to the water equivalent path length in energy-loss pCT. Reconstructing $\delta$ from $G$ is a typical tomography problem.

\subsection{Image reconstruction along most likely paths}

The true trajectory of a proton through the imaged object is unknown due to the stochastic nature of multiple Coulomb scattering, but the most likely path (MLP) can be estimated using well-established methods (Williams, 2004; Li et al., 2006; Schulte et al., 2008; Collins-Fekete et al., 2017; Krah et al., 2019).

In particular, we assume a point source moving on a circular trajectory around the isocenter. For a given source position, i.e. projection angle, we define the orthonormal basis vectors $\vec{u}, \vec{v}, \vec{w}$, where $\vec{w}$ defines the source-to-isocenter direction and $\vec{u}$ and $\vec{v}$ represent the axes of the detector plane. We denote the MLP of the $i$-th proton with $\vec{\Gamma}_{i}$ and describe it as function of the depth $w$ along $\vec{w}$, i.e. $\vec{\Gamma}_{i}(w) \cdot \vec{w}=w$, as do all MLP models.

In this notation, equation 8 formally becomes

$$
G\left(A_{\text {out }, i}\right)=\int_{0}^{A_{\text {out }, i}} \frac{1}{\tau_{w}(A)} \mathrm{d} A=\int_{\vec{\Gamma}_{i}} \delta\left(\vec{\Gamma}_{i}(w)\right) \mathrm{d} w .
$$

It relates the relative scattering power integrated along the MLP of the $i$-th proton to the water equivalent scattering thickness of the object along this MLP. It is worth noting a fundamental difference here however: while in energy-loss pCT, an energy is measured for each proton, the angular dispersion $A_{\text {out }}$ can only be estimated from a set of proton histories. In practice, the quantity $G\left(A_{\text {out }, i}\right)$ is thus not available proton per proton, as the equation suggests, but only for groups of protons. Estimation of $A_{\text {out }}$ (and $G\left(A_{\text {out }}\right)$ ) occurs when binning the data, as we will explain in the following.

We use the distance-driven binning technique initially proposed for energy-loss pCT (Rit et al., 2013) and later adapted for attenuation pCT (Quiñones et al., 2016). The basic idea of this method is to bin the measured data into a series of virtual projections at different distances from the sources while accounting for the MLP. The subsequent filtered backprojection (FBP) uses for each voxel the projection images which most appropriately matches the voxel's position in the image. In the following, we adapt the method to scattering $\mathrm{pCT}$. 
First off, let $\boldsymbol{j} \in \boldsymbol{J} \subset \mathbb{Z}^{2}$ be a set of spatial indices corresponding to the binning grid of a virtual projection and $\boldsymbol{y} \in \mathbb{R}^{2}$ the spatial location in the projection plane. Then the pixel indicator function $h: \mathbb{R}^{2} \rightarrow \mathbb{R}$ is defined as

$$
h_{\boldsymbol{j}}(\boldsymbol{y})= \begin{cases}1 & \text { if } \boldsymbol{y} \in \mathbb{R}^{2} \text { is in pixel } \boldsymbol{j} \\ 0 & \text { otherwise. }\end{cases}
$$

The measured quantity of interest is the difference between the entrance and exit direction of each proton, $\Delta \theta_{i}=\theta_{i}^{\text {out }}-\theta_{i}^{\text {in }}$. We treat MCS in the two directions perpendicular to the beam as independent statistical processes (Schulte et al., 2008) and use the average of the squared difference of both projected angles, $\left[\left(\Delta \theta_{i}^{u}\right)^{2}+\left(\Delta \theta_{i}^{v}\right)^{2}\right] / 2$, to estimate the variance attributed to a pixel. The distance-driven variance $\tilde{A}_{j}(w)$ of the angular distribution of protons crossing pixel $\boldsymbol{j}$ is defined as,

$$
\tilde{A}_{\boldsymbol{j}}(w)=\frac{\sum_{i \in I} h_{\boldsymbol{j}}\left(u_{i}(w), v_{i}(w)\right)\left[\left(\Delta \theta_{i}^{u}\right)^{2}+\left(\Delta \theta_{i}^{v}\right)^{2}\right] / 2}{\sum_{i \in I} h_{\boldsymbol{j}}\left(u_{i}(w), v_{i}(w)\right)},
$$

where we have used the fact that $\Delta \theta=0$ on average. The tilde symbol indicates that $\tilde{A}$ is only an estimate of the variance $A$.

The distance-driven binned variances $\tilde{A}_{\boldsymbol{j}}(w)$ are converted into scattering WEPL $G$ through an empirical lookup table. In particular, we ran a Monte Carlo simulation in GATE/Geant4 at high statistics $\left(10^{6}\right.$ primaries) of a perfectly collimated $\left(\theta_{\text {in }}=0\right)$ pin-like proton beam impinging onto boxes of water with varying thickness and recorded each proton's exit angle. For each thickness, we estimated the variance $A_{\text {out }}=\left\langle\theta_{\text {out }}^{2}\right\rangle$ and obtained the numerical relationship $G_{\mathrm{LUT}}\left(A_{\text {out }}\right)$ by interpolation. The distance-driven binned scattering WEPL is then

$$
g_{j}(w)=G_{\mathrm{LUT}}\left(\tilde{A}_{\boldsymbol{j}}(w)\right)
$$

A distance-driven projection $g_{j}^{\mathrm{s}}(w)$ is computed for each source position $s$ and the $\delta$-map is reconstructed using the same algorithm as in (Rit et al., 2013), i.e. using the FDK algorithm (Feldkamp et al., 1984) adapted with a voxel-specific backprojection selecting the distance $w$ according to the distance between the voxel and the exit detector for each source position.

\subsection{Dependence of relative scattering power on angular variance}

To which extent the reconstructed $\delta$-map reflects the material specific local properties (to be specified further down) is related to how much $\delta(z, A(z))$ depends on $A$. We recall that the same kind of argument holds for energy-loss where the relative stopping power is approximately independent of $E$ (Arbor et al., 2015). To study this aspect, we analysed the relation $\delta(A)$ numerically in different kinds of media. For computational simplicity, we relied on an analytical model rather than recurring to full Monte Carlo simulations.

In particular, we used the analytical model of MCS proposed by Highland (1975) and modified by Gottschalk et al. (1993) which accounts for the protons' energy loss while traversing an object. The variance $A(z)$ of the angular distribution in some depth $z$ in a homogeneous medium of radiation length $X_{0}$ is given by

$$
A(z) \equiv\langle\theta\rangle^{2}(z)=\left(1+\frac{1}{9} \log _{10} \frac{z}{X_{0}}\right)^{2} \Omega_{0}^{2} \int_{0}^{z} \frac{1}{p^{2}\left(z^{\prime}\right) v^{2}\left(z^{\prime}\right)} \frac{1}{X_{0}} \mathrm{~d} z^{\prime},
$$

with $\Omega_{0}=14.1 \mathrm{MeV}$ an empirical constant and $p$ and $v$ the protons' momentum and velocity, respectively. We note that several other analytical MCS models exist and refer to Gottschalk (2010) for a review. 
Evaluating the term

$$
\frac{1}{p^{2}(z) v^{2}(z)}=\frac{\left(E(z)+E_{p}\right)^{2} c^{2}}{\left(E(z)+2 E_{p}\right)^{2} E^{2}(z)}
$$

with $E_{p}$ the proton's rest energy, requires the kinetic energy $E(z)$ as a function of depth. This can be readily obtained numerically from a simple Monte Carlo simulation of protons penetrating the medium of interest or by numerically integrating tabulated stopping powers, e.g. from NIST's PSTAR database (Berger et al., 2005), in the continuous slowing down approximation.

The scattering power, i.e. the derivative of equation 13, contains two terms,

$$
\begin{aligned}
T(z)=\frac{\mathrm{d} A}{\mathrm{~d} z}(z) & =\left(1+\frac{1}{9} \log _{10} \frac{z}{X_{0}}\right)^{2} \Omega_{0}^{2} \frac{1}{p^{2}(z) v^{2}(z)} \frac{1}{X_{0}} \\
& +\frac{2}{9 z}\left(1+\frac{1}{9} \log _{10} \frac{z}{X_{0}}\right) \Omega_{0}^{2} \int_{0}^{z} \frac{1}{p^{2}\left(z^{\prime}\right) v^{2}\left(z^{\prime}\right)} \frac{1}{X_{0}} \mathrm{~d} z^{\prime} \\
& \approx\left(1+\frac{1}{9} \log _{10} \frac{z}{X_{0}}\right)^{2} \Omega_{0}^{2} \frac{1}{p^{2}(z) v^{2}(z)} \frac{1}{X_{0}}
\end{aligned}
$$

The second term stems from the chain rule applied to the logarithmic pre-factor and its magnitude is on the order of $1-3 \%$ of the first term. We neglect it in the following.

The relationship $\tau(A)$ for a given medium can be constructed numerically by evaluating equations 13 and 15 and linking $T$ and $A$ by interpolation via $z$. The relative scattering power of the medium is $\delta(A)=\tau(A) / \tau_{w}(A)$.

At $z=0$ (i.e. $A=0$ ) in equation 15 , the relative scattering power $\tau(0) / \tau_{w}(0)=T(0) / T_{w}(0)$ corresponds to the radiation length ratio $X_{0, w} / X_{0}$. This would also be true, if the energy-loss in any medium were the same as in water and the energy-dependent terms $1 / \beta^{2} p^{2}$ cancelled out (disregarding the logarithmic pre-factor). Therefore, we use the ratio $X_{0, w} / X_{0}$ as material specific property to compare relative scattering powers $\delta$ with.

We use will the expressions presented in this section to analyse how much $\delta$ depends on $A$ in materials and beam energies of interest for scattering proton $\mathrm{CT}$.

\subsection{Statistical limitations in scattering proton CT}

In this section, we derive an expression to quantify the inherent noise of a scattering pCT image and compare it to energy loss pCT. We disregard noise and artefacts created by the discretization of the reconstruction algorithm and concentrate on noise due to uncertainties in the projections only. For simplicity, we consider a homogeneous water cylinder and the noise at its centre. We tacitly assume that all expressions below are evaluated in the central pixel $\boldsymbol{j}_{\text {centre }}$ of a projection plane at depth $w_{\text {centre }}$ corresponding to the cylinder centre as Schulte et al. (2005).

As part of the distance driven binning, we estimate the angular variance $A$ from a finite number of proton histories as $\tilde{A}=(1 / 2 N) \sum_{i \in I}\left[\left(\Delta \theta_{i}^{u}\right)^{2}+\left(\Delta \theta_{i}^{v}\right)^{2}\right]$ (see equation 11), where $N$ is the number of proton histories in a pixel. This estimate will be intrinsically uncertain even if the angles $\Delta \theta$ are measured with perfect accuracy. The standard error $\sigma_{\tilde{A}}^{2}$ of the variance $\tilde{A}$ estimated from a finite sample of the distribution is

$$
\sigma_{\tilde{A}}^{2}=\frac{1}{2 N}\left(\mu_{4}-\frac{2 N-3}{2 N-1} A^{2}\right) \approx \frac{1}{2 N}\left(\left[3-\frac{2 N-3}{2 N-1}\right] A^{2}\right),
$$

where $\mu_{4}$ is the fourth moment of the distribution of $\Delta \theta$ and $A$ its variance (Rao, 1973). The normalisation factor is $1 / 2 N$ instead of $1 / N$ because it accounts for the fact that $\Delta \theta^{u}$ and $\Delta \theta^{v}$ are both used and that they are independent measurements. As the true values of $A^{2}$ and $\mu_{4}$ are unknown, we estimate them from a high-statistics Monte Carlo simulation of protons penetrating 
a water phantom. The simplified expression on the right-hand side is valid under the assumption that the angles $\Delta \theta$ are normally distributed $\left(\mu_{4}=3 A^{2}\right)$. This is only approximately true because of large angle electromagnetic scattering leading to non-Gaussian tails (Gottschalk, 2010) and due to nuclear interactions present in measured data if their filtering is imperfect.

Following the work of Schulte et al. (2005) on the intrinsic noise in energy-loss pCT, we use first-order error propagation to calculate the uncertainties of the scattering WEPL,

$$
\sigma_{g}^{2}=\sigma_{\tilde{A}}^{2}\left(\left.\frac{\mathrm{d} G}{\mathrm{~d} A}\right|_{A=\tilde{A}_{\text {out }}}\right)^{2}=\frac{\sigma_{\tilde{A}}^{2}}{\tau_{w}^{2}\left(\tilde{A}_{\text {out }}\right)},
$$

where we have used the fact that $G$ is the primitive function of $A$ by construction (see equation 9 ).

The effect of the ramp filter on the uncertainty $\sigma_{g}^{2}$ in the central pixel $\boldsymbol{j}_{\text {centre }}$ in the projections can be described by a factor $1 / 12 a^{2}$ (Gore et al., 1978) and reconstructing the image via FBP involves halving the sum of the $P$ projections distributed over the full $2 \pi$ circle (in our case) in constant angular increments $\Delta \alpha=2 \pi / P$, so that

$$
\sigma_{\delta}^{2}=\frac{1}{2} \sum_{p=1}^{P} \frac{1}{12 a^{2}} \sigma_{g}^{2} \Delta \alpha^{2}=\sigma_{g}^{2} \frac{\pi^{2}}{6 a^{2} P},
$$

where we have used the fact that $\sigma_{g}^{2}$ is independent of the projection angle in cylindric objects. Combining equations 17,18 , and 19 , we obtain

$$
\sigma_{\delta}^{2}=\frac{1}{2 N}\left(\tilde{\mu}_{4}-\frac{2 N-3}{2 N-1} \tilde{A}_{\text {out }}^{2}\right) \frac{1}{\tau_{w}^{2}\left(\tilde{A}_{\text {out }}\right)} \frac{\pi^{2}}{6 a^{2} P} .
$$

We wish to express this uncertainty as a function of dose to the centre of the cylinder along the lines of Schulte et al. (2005). To this end, it is useful to introduce the proton fluence into equation 20 above. Let $\Phi_{\text {in }}$ be the initial fluence of a proton beam impinging centrally onto the cylinder, then the downstream fluence behind the cylinder centre and the fluence at the centre, respectively, are

$$
\begin{aligned}
\Phi_{\text {out }} & =\Phi_{\text {in }} \exp (-2 \kappa r) \\
\Phi_{\text {centre }} & =\Phi_{\text {in }} \exp (-\kappa r),
\end{aligned}
$$

where $r$ is the cylinder radius and $\kappa$ the attenuation coefficient due to nuclear interactions. Here, we approximate proton trajectories as straight lines disregarding MCS. The number $N$ in equation 20 refers to the number of protons crossing an area $a \times a$ in the object's centre given by the pixel side length and the slice thickness (we use cubic voxels) and exiting the object downstream, so that

$$
\frac{N}{a^{2}}=\Phi_{\text {out }}=\Phi_{\text {centre }} \exp (-\kappa r) .
$$

Inserting equation 23 into equation 20 and letting $(2 N-3) /(2 N-1) \approx 1$ (because $N \gg 1$ ), we have

$$
\sigma_{\delta}^{2}=\frac{\left(\tilde{\mu}_{4}-\tilde{A}_{\text {out }}^{2}\right) \pi^{2}}{\tau_{w}^{2}\left(\tilde{A}_{\text {out }}\right) 12 P a^{4} \Phi_{\text {centre }} \exp (-\kappa r)} .
$$

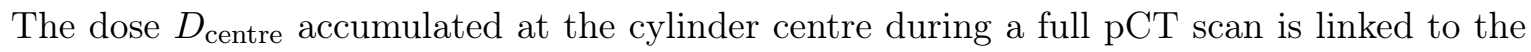
fluence $\Phi_{\text {centre by }}$

$$
D_{\text {centre }}=P \Phi_{\text {centre }}\left[\frac{S\left(E_{\text {centre }}\right)+\kappa \gamma E_{\text {centre }}}{\rho}\right]
$$


where $S\left(E_{\text {centre }}\right)$ is the proton stopping power for the proton energy at the centre, $\rho$ is the mass density of the cylinder material and $\gamma=0.65$ is the fraction of energy transferred to secondary particles during nuclear interactions (Schulte et al., 2005). Substituting equation 25 into equation 24 yields the final expression:

$$
\sigma_{\delta}=\frac{\left(\tilde{\mu}_{4}-\tilde{A}_{\text {out }}^{2}\right)^{1 / 2}}{\tau_{w}\left(\tilde{A}_{\text {out }}\right)}\left[\frac{\pi^{2}\left(S\left(E_{\text {centre }}\right)+\kappa \gamma E_{\text {centre }}\right)}{12 a^{4} \exp (-\kappa r) \rho D_{\text {centre }}}\right]^{1 / 2} .
$$

If the angles $\Delta \theta$ are normally distributed, $\tilde{\mu}_{4}-\tilde{A}_{\text {out }}^{2}=2 \tilde{A}_{\text {out }}^{2}$, and the equation above simplifies accordingly.

For comparison, we state the analogous expression for the uncertainty of the reconstructed relative electron density (proportional to RSP) in energy-loss pCT as derived in Schulte et al. (2005) (here with an additional factor 2 in the denominator to account for halving opposite projections along a full 360 degree acquisition trajectory):

$$
\sigma_{\eta_{e}}=\frac{\sigma_{\mathrm{E}_{\text {out }}}}{S\left(E_{\text {out }}\right)}\left[\frac{\pi^{2}\left(S\left(E_{\text {centre }}\right)+\kappa \gamma E_{\text {centre }}\right)}{6 a^{4} \exp (-\kappa r) \rho D_{\text {centre }}}\right]^{1 / 2}
$$

In a water cylinder with $10 \mathrm{~cm}$ radius, $E_{\text {centre }} \approx 151 \mathrm{MeV}$ for a $200 \mathrm{MeV}$ proton beam and $\approx 263 \mathrm{MeV}$ for a $300 \mathrm{MeV}$ proton beam. We use the same value $\kappa=0.01 \mathrm{~cm}^{-1}$ as Schulte et al. (2005) which is inferred from proton on oxygen reactions (Malmer, 2001). At $151 \mathrm{MeV}$, the proton stopping power $S\left(E_{\text {centre }}\right)$ in water is about $5.4 \mathrm{MeV} \mathrm{cm}^{2} / \mathrm{g}$ (Berger et al., 2005) and the scattering power $T_{w}$ is about $6.5 \times 10^{-5} \mathrm{~cm}^{-1}$. At $263 \mathrm{MeV}$, the values are about $3.8 \mathrm{MeV} \mathrm{cm} / \mathrm{g}$ and $2.3 \times 10^{-5} \mathrm{~cm}^{-1}$, respectively.

\subsection{Monte Carlo simulations}

We performed Monte Carlo simulations using Geant4 (Agostinelli et al., 2003) version 10.01.p02 via GATE (Sarrut et al., 2014) to evaluate the various aspects of scattering proton CT described in the preceding sections. We used the QGSP_BIC physics list, but retained only those protons for the reconstruction which had not undergone any nuclear interaction. Angular deflection in our data is therefore purely caused by MCS. On experimental data, one would impose a threshold-based filtering, commonly referred to as "3-sigma" cuts, to eliminate events with large angular deviation which are likely to be associated with nuclear interactions (Schulte et al., 2008). Furthermore, we set the proton step size limit to $1 \mathrm{~mm}$ throughout this study.

\subsubsection{Phantoms}

We used a homogeneous water cylinder with $20 \mathrm{~cm}$ diameter to study the noise properties of scattering proton $\mathrm{CT}$ and a homogeneous aluminium cylinder of $5 \mathrm{~cm}$ diameter to illustrate how the dependence of $\delta$ on $A$ (see section 2.4) results in a cupping artefact. We selected the Gammex 467 phantom (Sun Nuclear, Melbourne, USA) which has a diameter of $33 \mathrm{~cm}$ and includes 17 cylindrical tissue equivalent inserts. Finally, we employed a cylindrical water phantom with cylindrical aluminium inserts of $1.5 \mathrm{~cm}$ diameter arranged in a spiral around the centre (see figure 8 in the Results section). All phantoms were implemented digitally in GATE by specifying their material composition and dimensions.

For the part of our study based on analytical models, we used a $20 \mathrm{~cm}$ thick block of water with a $2 \mathrm{~cm}$ thick slab of bone-like material (SB3 Gammex insert material) at variable depths. This phantom was described analytically by its RSP and radiation length $X_{0}$ as a function of depth. 


\subsubsection{Simulation set-up}

The simulation set-up consisted of a mono-energetic fan-beam proton source, the phantom at isocentre, and two trackers (one upstream and one downstream of the phantom). The fan-beam source was positioned $5 \mathrm{~m}$ upstream from isocentre and its dimensions at isocentre were adapted to the phantom size: $267 \times 5 \mathrm{~mm}^{2}$ for the water, aluminium, and spiral phantom, and $377 \times 5 \mathrm{~mm}^{2}$ for the Gammex phantom, where the longer dimension corresponds to the transverse direction and the shorter one to the direction of the rotation axis. The beam energy was either $200 \mathrm{MeV}$ or $300 \mathrm{MeV}$, as we will indicate in the Results section. The uniform source fluence was set to yield a dose at the phantom centre of $50 \mathrm{mGy}$ for an entire scan, except for the water cylinder phantom where the dose varied as indicated in the Results section. The ideal trackers recorded each proton's position and direction and the down-stream trackers additionally registered each proton's energy for the energy-loss images. A total of 360 projections were acquired in angular increments of 1 degree and binned into $1 \times 1 \mathrm{~mm}^{2}$ pixels for the water, aluminium, and Gammex phantom, and a finer $0.5 \times 0.5 \mathrm{~mm}^{2}$ pixels for the spiral phantom which was used to assess spatial resolution.

\subsection{Image quality metrics}

Spatial resolution Following Khellaf et al. (2020), we estimated the spatial resolution using the spiral phantom described in section 2.6.1. In and around each aluminium insert, we collected the pixel values together with their distance to the insert centre, $r$. This provided us with an isotropically sampled radial edge spread function (ESF), as in Richard et al. (2012), to which we fitted the following model based on the error function (erf):

$$
\operatorname{ESF}(r)=\frac{a}{2}\left[1-\operatorname{erf}\left(\frac{r-R}{\sqrt{2} \sigma}\right)\right]+1,
$$

with $a, R$, and $\sigma$ fit parameters. The rationale of using the error function is that image blur in proton $\mathrm{CT}$ is dominated by MCS which in turn is approximately Gaussian. As measure for the spatial resolution (in line pairs per $\mathrm{mm}$ : $\mathrm{lp} / \mathrm{mm}$ ), we used the frequency $f_{10 \%}$ at which the modular transfer function of the ESF decayed below $10 \%$ of its maximum value, which is analytically related to $\sigma$,

$$
f_{10 \%}=\sqrt{\frac{\ln 10}{2}} \frac{1}{\pi \sigma} .
$$

Noise We determined image noise as the variance of the central pixel value over 110 reconstructed images of the cylindrical water phantom, each based on independently simulated Monte Carlo data. We used this value as reference to compare equation 26 with.

Contrast-to-noise and signal-to-noise We calculated the contrast-to-noise ratio (CNR) and signal-to-noise ratio (SNR) of the inserts in the Gammex phantom (see section 2.6.1). Specifically, we defined a circular region-of-interest (ROI) of half the insert diameter and determined the CNR and SNR as:

$$
\begin{aligned}
\mathrm{SNR} & =\frac{\mu_{\mathrm{ROI}}}{\sigma_{\mathrm{ROI}}} \\
\mathrm{CNR} & =\frac{\mu_{\mathrm{ROI}}-\mu_{\mathrm{water}}}{\sigma_{\mathrm{ROI}}}
\end{aligned}
$$

where $\mu_{\mathrm{ROI}}$ and $\sigma_{\mathrm{ROI}}$ are the mean value and root-mean-square-error per ROI, and $\mu_{\text {water }}$ the mean value of the water-like base structure of the phantom. 


\section{Results}

\subsection{Dependence of relative scattering power on $\mathrm{A}$}

We analysed the dependence of relative scattering power $\delta$ on angular variance $A$ as explained in section 2.4. Figure 1 condenses much of the relevant information: the upper four panels (a-d) for a proton energy of $200 \mathrm{MeV}$ and the lower four (e-h) for $300 \mathrm{MeV}$. The analysis was done in homogeneous material descriptions corresponding to the different insert materials of the Gammex phantom (colour-coded). The dash-dotted lines refer to liquid water.

Panels a and e show the angular variance $A(z)$ of an initially co-linear pin-like proton beam as a function of depth $z$ and panels $\mathrm{b}$ and $\mathrm{f}$ the scattering power $T(z)$. Notably, $A(z)$ and $T(z)$ become very steep and non-linear towards the protons' range. This obviously corresponds to shallower depths at $200 \mathrm{MeV}$ than at $300 \mathrm{MeV}$. Panels $\mathrm{c}$ and g show the scattering power $\tau(A)$ strongly varies as a function of $A$. Panels $\mathrm{d}$ and $\mathrm{h}$ show the relative scattering power $\delta(A)$ as a function of $A$. For materials with a density similar or inferior to water, this dependence is relatively weak, while $\delta$ decreases considerably with increasing $A$ for bone-like tissues (CB and SB). For example, the relative scattering power of cortical bone (CB2_50) at a variance of $1.5 \times 10^{-3} \mathrm{rad}^{2}$ (representative of $20 \mathrm{~cm}$ of water at $200 \mathrm{MeV}$ proton energy), is about $20 \%$ lower than its $X_{0, w} / X_{0}$ ratio.

The left panel of figure 2 shows the reconstructed image of the aluminium cylinder and the right panel depicts the radial profile of reconstructed values. There is a clear cupping artefact: relative scattering power decreases towards the centre of the cylinder while it reaches about the characteristic ratio of $X_{0, w} / X_{0}=4.06$ for aluminium near the edges. The fact that central pixels are associated with comparatively large angular variances for which $\delta$ is more strongly underestimated explains this observation. We remark that the mass density of aluminium $\left(2.7 \mathrm{~g} / \mathrm{cm}^{3}\right)$ is about 1.5 times higher than in the most dense bone insert (SB3), making the cupping artefact particularly pronounced.

Figure 3 shows the analytically calculated relative scattering power in a case where protons traverse the water phantom with bone slab inserts (section 2.6.1) for a proton energy of $200 \mathrm{MeV}$ and $300 \mathrm{MeV}$. To this end, we made the radiation length in equations 13 and 15 a function of depth, $X_{0}(z)$. The dashed coloured lines indicate the average value of $\delta$ in the slabs which are systematically lower than the ratio $X_{0, w} / X_{0}$ (black dashed line). The presence of the slabs also causes an underestimation of $\delta$ in the water subsequently traversed. At $200 \mathrm{MeV}$, the degree of underestimation varies considerably depending on the depth of the bone slab, while it is almost independent of the insert depth at $300 \mathrm{MeV}$.

\subsection{Image quality: noise, SNR, CNR}

As a figure of merit for the image noise, we calculated the expected precision of the reconstructed relative scattering power in the central pixel of a water cylinder, as explained in section 2.5 (equation 26). We compare the results with the relative stopping power precision in energy-loss proton CT employing the formalism of Schulte et al. (2005) (our equation 27).

The left panel in figure 4 shows the precision as a function of cylinder diameter for a dose of 10 mGy at the cylinder centre. The right panel depicts how relative scattering power precision varies as the inverse square root of the dose. For comparison, we included the intrinsic noise in attenuation proton CT in an otherwise identical setting, as derived in Quiñones et al. (2016).

Regardless of this, the most striking conclusion from our figure 4 is that the order of magnitude in precision is vastly different in the two modalities (beware the two separate y-axes): relative stopping power precision is about $1.6 \%$ for a $20 \mathrm{~cm}$ cylinder while it is about $50 \%$ in scattering proton CT. This was also visually apparent in the Gammex and spiral phantom images in figures 6 and 8 , respectively. The main reason for this is that estimating the variance of a distribution (in scattering proton $\mathrm{CT}$ ) is less certain than estimating the mean value (the mean residual energy 


\section{$200 \mathrm{MeV}$}
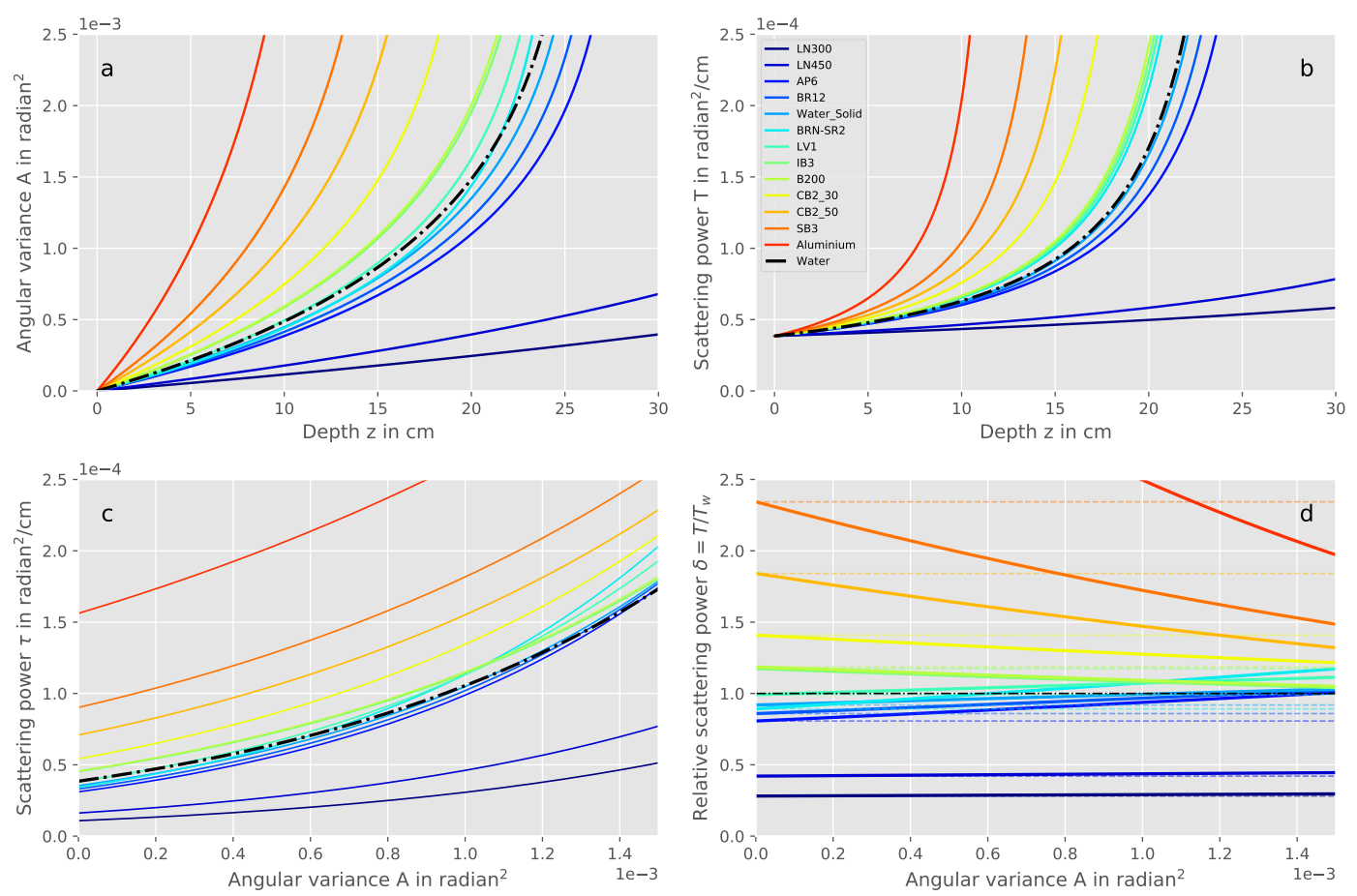

$300 \mathrm{MeV}$
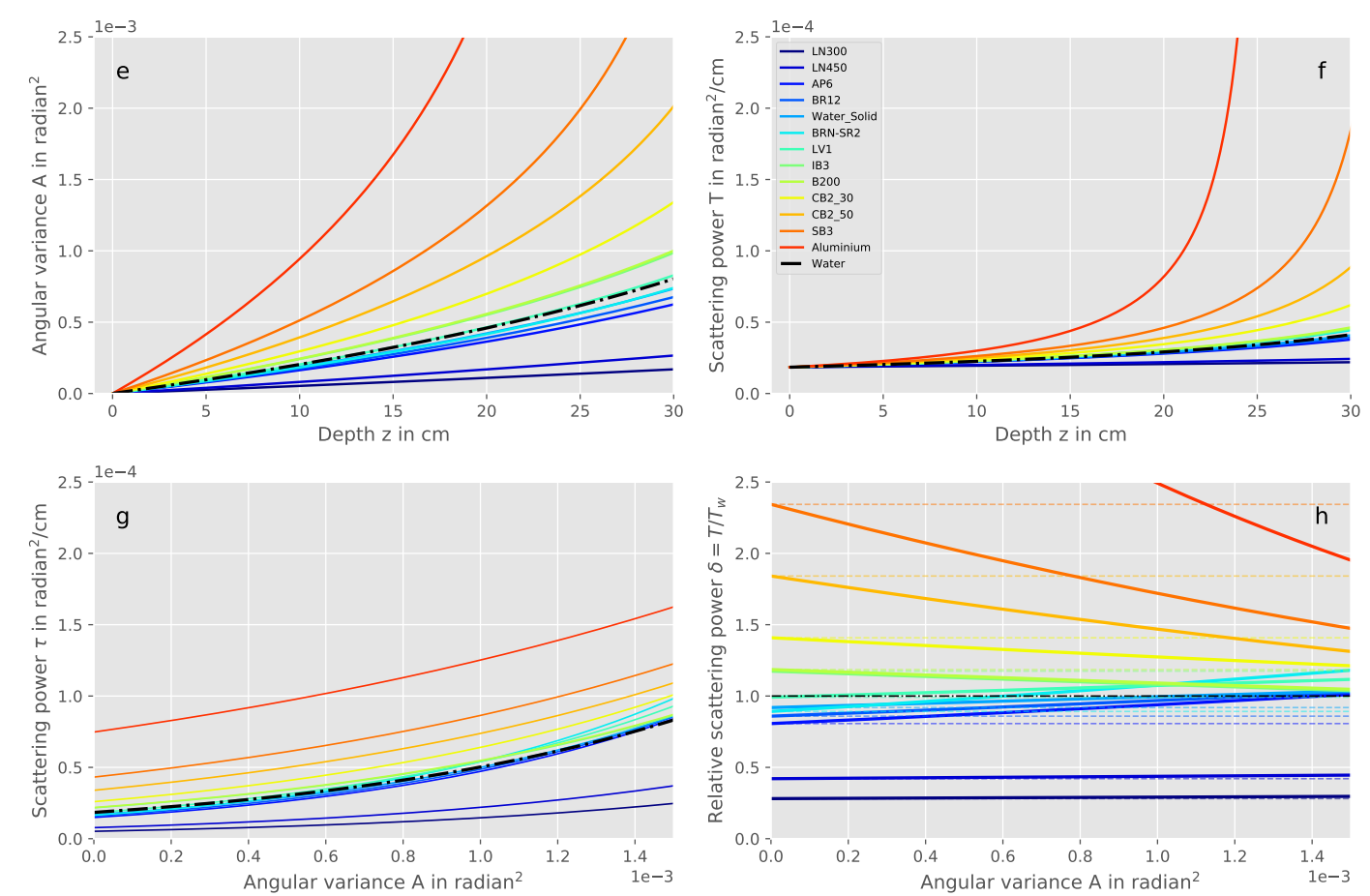

Figure 1: Relationships between angular variance, scattering power, and relative scattering power for $200 \mathrm{MeV}$ (upper group of panels) and $300 \mathrm{MeV}$ (lower panels). Coloured lines refer to Gammex insert materials; the black dash-dotted line always refers to water. Colored dashed lines in panels $\mathrm{d}$ and $\mathrm{h}$ indicate the radiation length ratio $X_{0, w} / X_{0}$. All plots were generated analytically as explained in section 2.4 . 

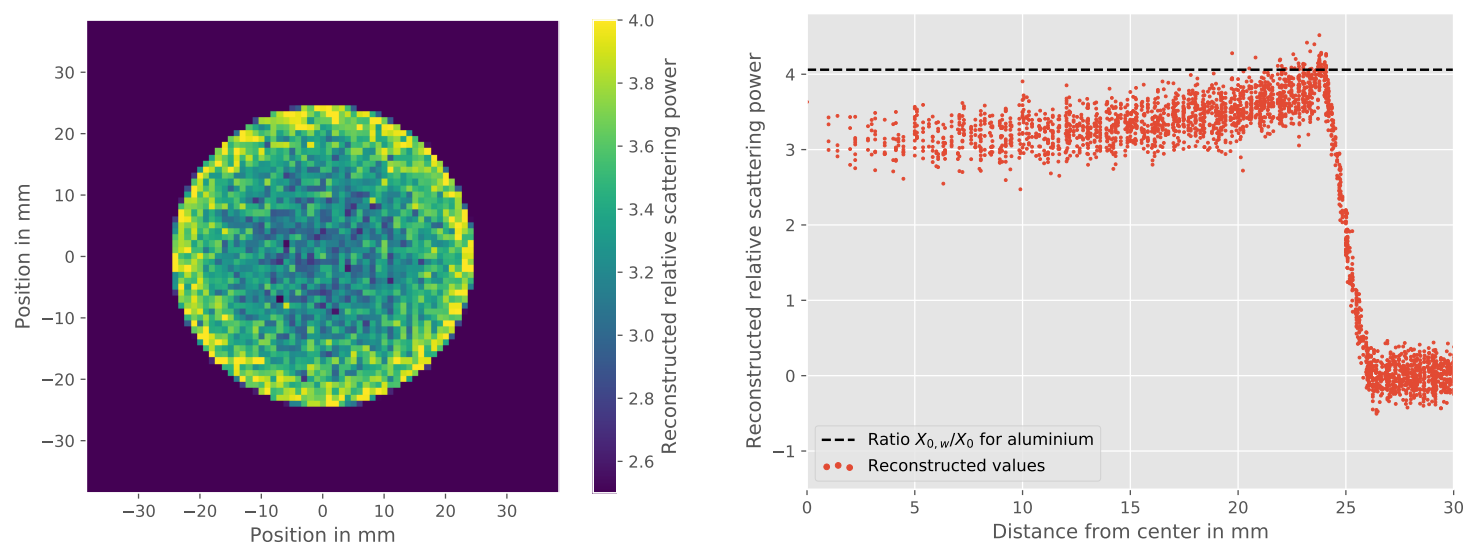

Figure 2: Left: Reconstructed relative scattering power image of the aluminium cylinder phantom. Right: Profile of relative scattering power as a function of distance from the cylinder centre. The interesting detail is the cupping artefact, i.e. the decrease of reconstructed values towards the centre.
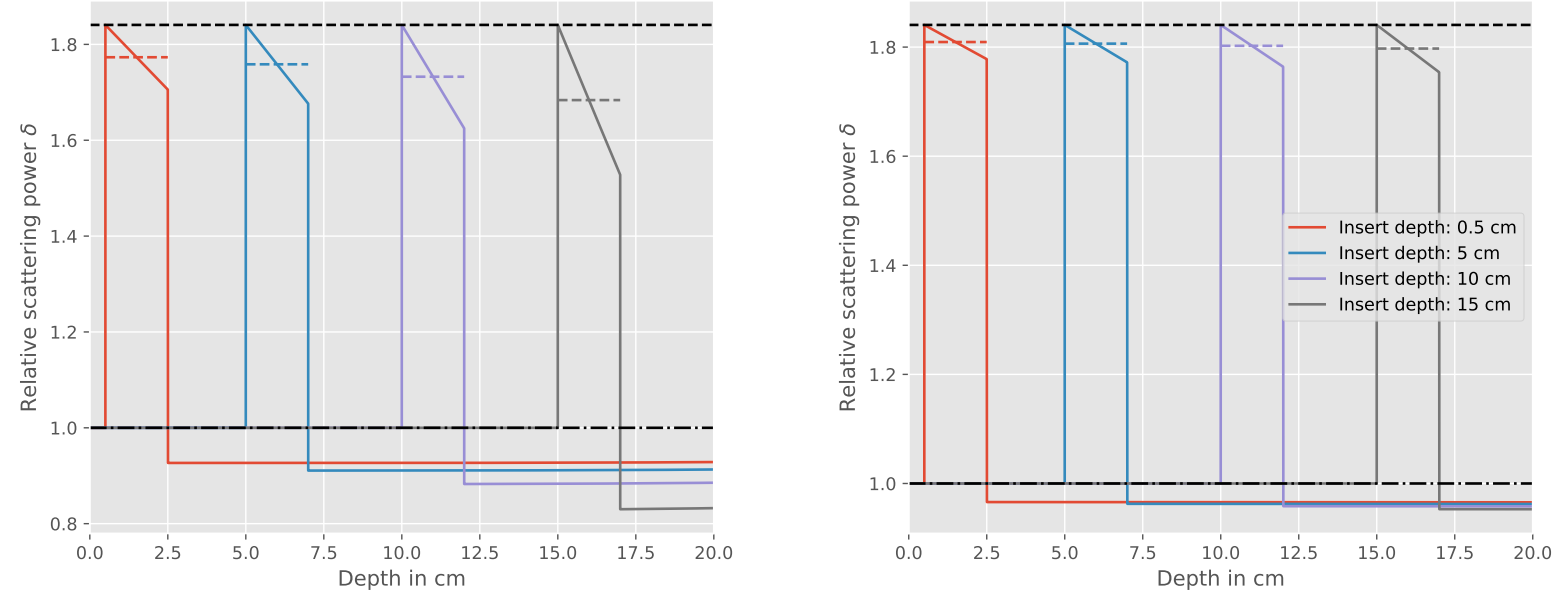

Figure 3: Relative scattering power as a function of depth in a $20 \mathrm{~cm}$ thick water phantom into which a bone-like slab of $2 \mathrm{~cm}$ is inserted at different depths. Beam energies: $200 \mathrm{MeV}$ (left) and $300 \mathrm{MeV}$ (right). 

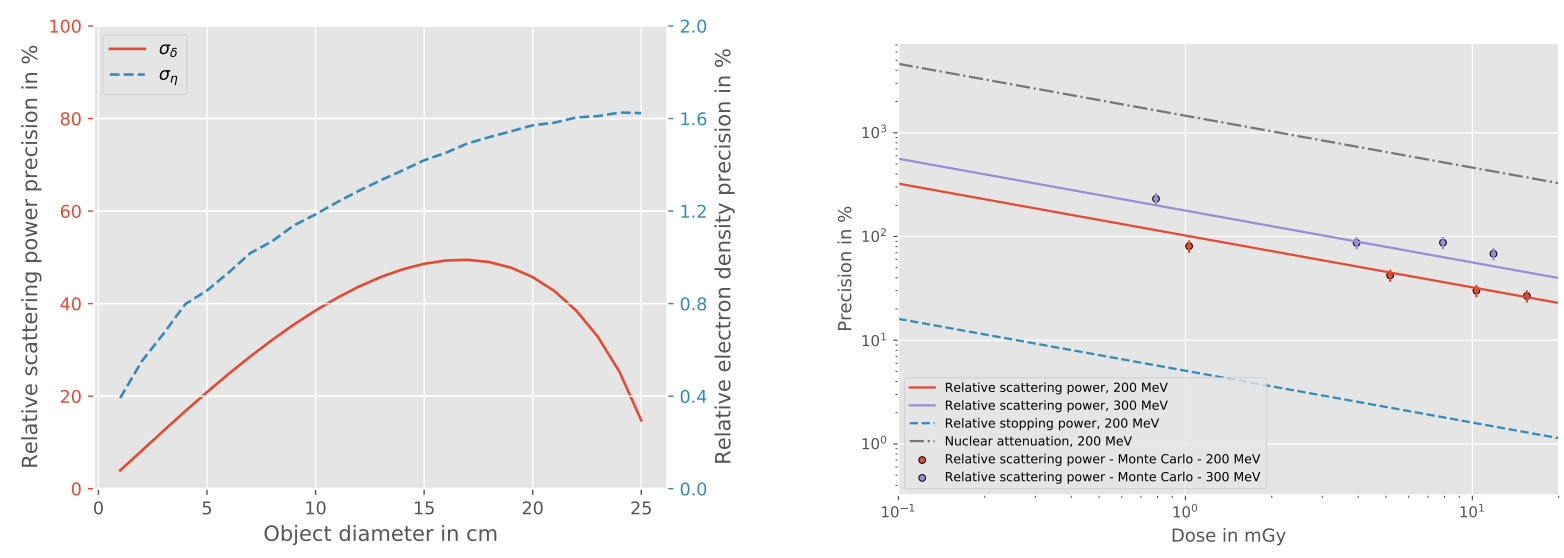

Figure 4: Left: Relative scattering/stopping power precision at the center of a water cylinder as a function of its diameter for scattering and energy-loss proton CT, respectively. Beware of separate y-axes. Right: Precision in a cylinder of $20 \mathrm{~cm}$ diameter as a function of dose at the cylinder centre for scattering (equation 26), energy-loss (equation 27), and attenuation proton CT (calculated as in Quiñones et al. (2016)).

in energy-loss proton CT). Specifically, the factor $\tilde{A}_{\text {out }} / \tau_{w}$ in equation 26 is 20-40 times larger than the factor $\sigma_{E_{\text {out }}} / S$ in equation 27 .

Figure 5 shows the CNR (upper panel) and SNR (centre panel) of the Gammex phantom inserts derived from the Monte Carlo simulated images shown in figure 6. The attenuation proton CT image was reconstructed as described in (Quiñones et al., 2016). We have arranged the values as a function of mass density to illustrate this dependence. For inserts with a mass density close to that of the water-like support structure of the Gammex phantom (most abundant in the human body), the CNR in scattering proton CT is below 0.5 and an order of magnitude lower than the CNR in energy-loss proton CT. The SNR is 3-4 in scattering proton CT compared to around 200 in energy-loss proton CT. Overall, both CNR and SNR are lower by a factor of 40-50 in scattering proton CT than in energy-loss proton CT. This is mainly due to the higher noise in the former modality and not due to intrinsically different levels of contrast, which can be seen by the following two observations: For a water cylinder of $33 \mathrm{~cm}$ diameter imaged with a beam energy of $300 \mathrm{MeV}$ at $50 \mathrm{mGy}$ dose at the centre, the $\delta$-resolution is about $100 \%$ while the RSP resolution is about $2.5 \%$ (values not shown in figure 4), i.e. a relative factor of 40 . On the other hand, the lower panel in figure 5 provides an indication of the intrinsic contrast in each modality showing $X_{0, w} / X_{0}$ and RSP, respectively. They are essentially identical except for bone-like densities where scattering proton CT promises $50 \%$ higher contrast.

\subsection{Accuracy of relative scattering power $\delta$}

Figure 7 shows the accuracy of the reconstructed relative scattering power (red) compared to the material specific ratio $X_{0, w} / X_{0}$ (black). The values were derived from the Monte Carlo simulated images shown in figure 6 by averaging over 330 pixels around the centre of each insert. The inserts are sorted according to their density. For bone-like inserts (IB3, B200, CB2_30, CB2_50, SB3), the reconstructed value of $\delta$ underestimates the ratio $X_{0, w} / X_{0}$. Instead, for the inserts AP6, BR_12, and BRN-SR2, the reconstructed $\delta$ overestimates $X_{0, w} / X_{0}$. This is in line with the observations made earlier in section 3.1 regarding figure 1 where $\delta(A)$ decreased as a function of $A$ for bone-like materials and increased for some of the others. The upper right panel in figure 8 shows the mean value of $\delta$ per insert (aluminium) in the spiral phantom as a function of distance from the phantom centre. The ratio $X_{0, w} / X_{0}$ is increasingly underestimated for inserts closer to the centre. This is expected because on average over all projections, the 

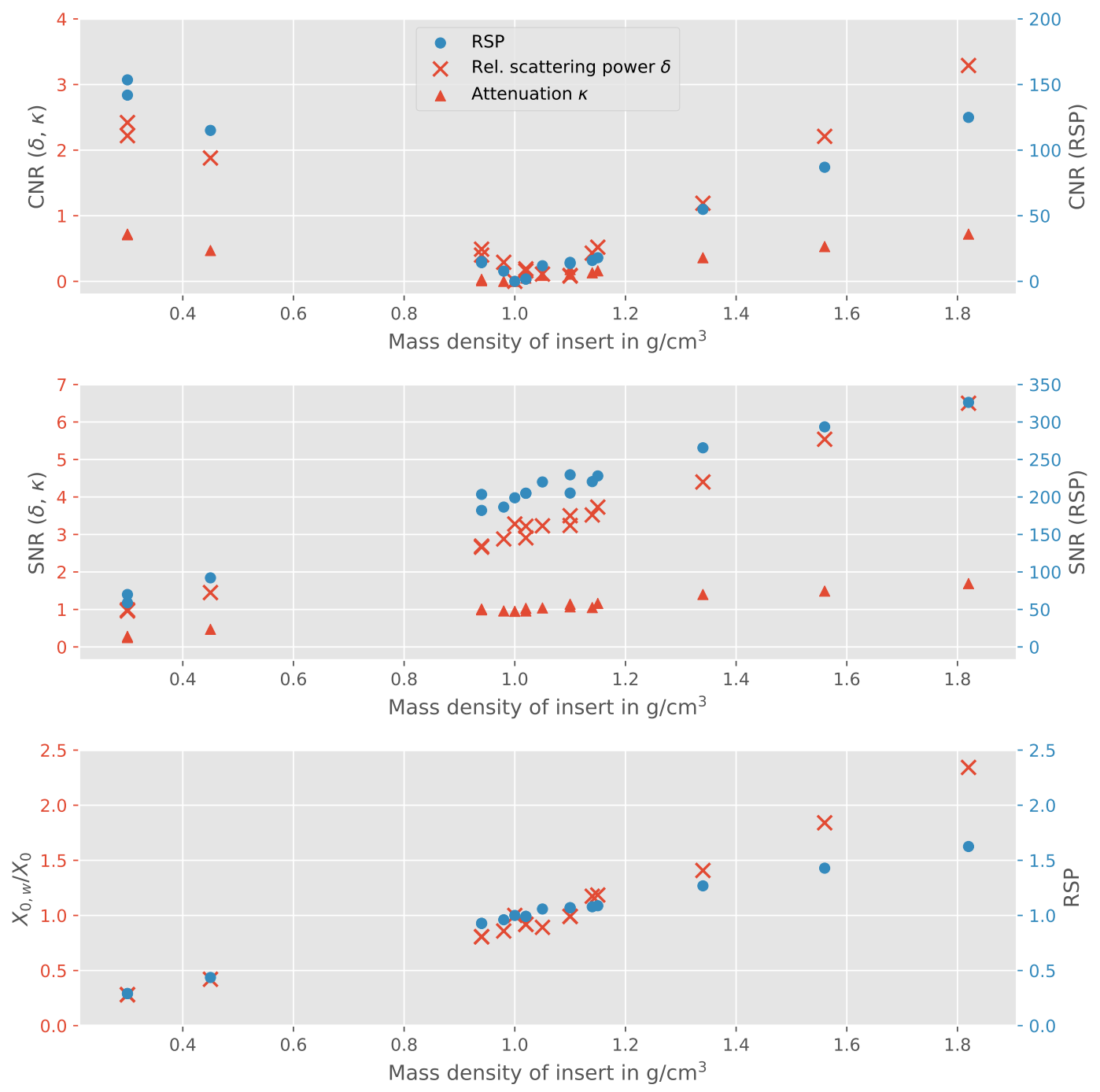

Figure 5: Upper and middle panels: CNR and SNR of the Gammex phantom inserts obtained via Monte Carlo simulation. Data are presented as a function of the insert density. Lower panel: Material specific radiation length ratio $X_{0, w} / X_{0}$ and RSP.
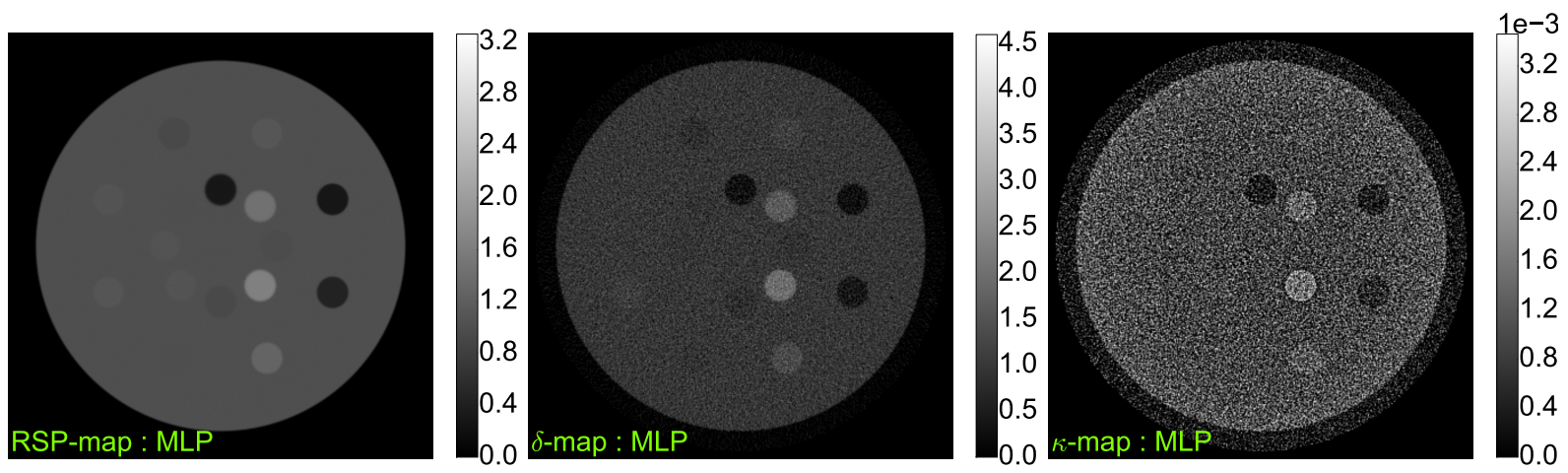

Figure 6: Reconstructed images of the Gammex phantom in energy-loss (left), scattering (centre), and attenuation (right) proton CT. Gray values range from zero to twice the value in the most dense insert (SB3). 


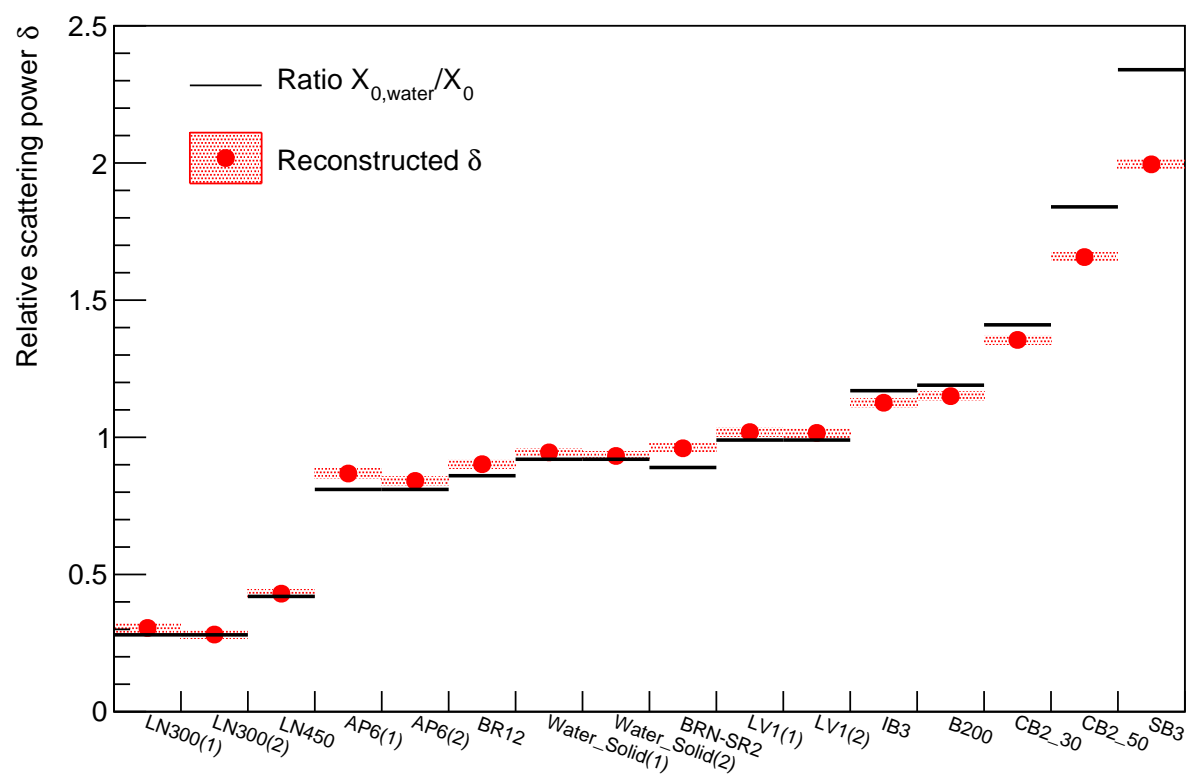

Figure 7: Mean relative scattering power $\delta$ values of the Gammex phantom inserts extracted from the reconstructed image shown in the centre panel in figure 6 (red dots). Red shaded areas indicate the standard error of the mean value. Black lines indicate the material specific radiation length ratio $X_{0, w} / X_{0}$.

variance $A$ is larger for central inserts than for peripheral ones so that the discrepancy between $\delta(A)$ and $X_{0, w} / X_{0}$ is larger.

\subsection{Spatial resolution}

The lower right panel in figure 8 shows the spatial resolution estimated from the spiral phantom images as a function of distance to the phantom centre. The values were obtained by fitting an error function to line profiles positioned radially around each insert as described in section 2.7. An example profile is shown in the centre right panel. Error bars were derived from the fit uncertainty. The plot compares scattering proton CT using distance-driven binning reconstruction, straight line reconstruction, and energy-loss proton $\mathrm{CT}$ with distance driven binning reconstruction.

Spatial resolution ranges from $0.7 \mathrm{lp} / \mathrm{mm}$ at the centre to $1.4 \mathrm{lp} / \mathrm{mm}$ near the edge. Incorporating the MLP improves spatial resolution by almost a factor 3. Spatial resolution in scattering proton CT appears to be slightly inferior compared to energy-loss proton CT near the phantom centre, although this difference could still be explained by the statistical uncertainty of the data.

\section{Discussion}

In this work, we investigated scattering proton CT reconstruction. Specifically, we introduced the relative scattering power in analogy to the relative stopping power in energy-loss proton CT and adapted a distance-driven filtered backprojection algorithm to scattering proton CT. It is worth mentioning that there is some resemblance between our algorithm and the noise reconstruction in Rädler et al. (2018).

Our results show that the reconstruction algorithm works well and incorporating the MLP in the backprojection improves spatial resolution by almost a factor of three compared to straight 

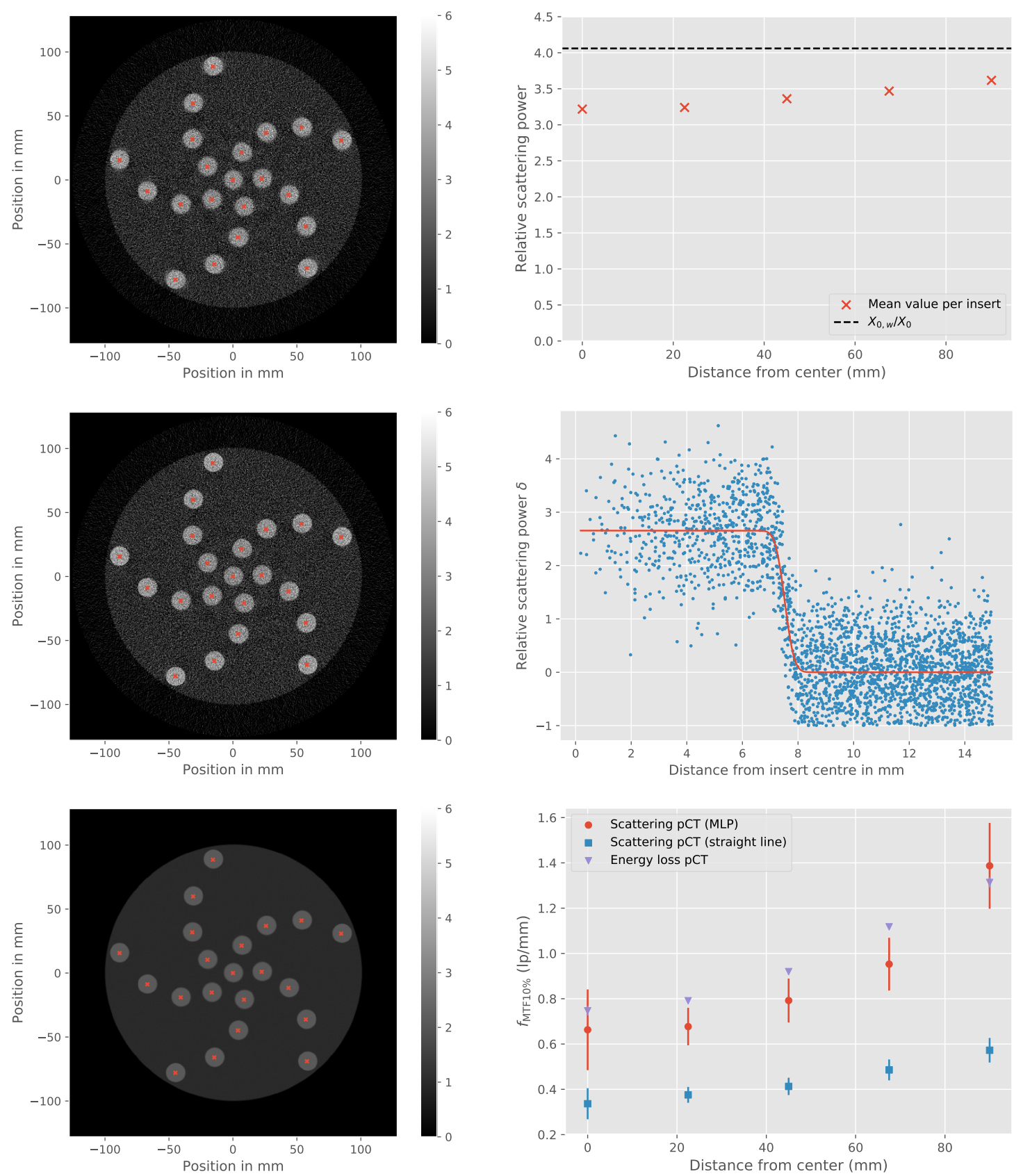

Figure 8: Left column: Reconstructed images of the spiral phantom: scattering proton CT in straight line and MLP reconstruction (upper and center panel, respectively) and energy-loss proton CT in MLP reconstruction. Right column: Mean reconstructed value as a function of insert distance from centre (upper panel), example radial profile across an insert edge (centre panel), and spatial resolution as a function of distance from the phantom centre (lower panel). 
line projection in the spiral phantom used in this work (figure 8). Spatial resolution was comparable to that in energy-loss proton CT (figure 8).

The rationale of introducing relative scattering power was to obtain a quantity which does not strongly vary as a function of angular variance. Our analysis based on tissue-like substitute materials showed that this is approximately true for some materials similar to or less dense than water. For denser bone-like materials, the relative scattering power decreases with increasing angular variance by up to $20 \%$ in a $20 \mathrm{~cm}$ thick phantom at $200 \mathrm{MeV}$ and up to about $10 \%$ at $300 \mathrm{MeV}$ (figure 1). In objects denser than water, such as an aluminium cylinder, this leads to a cupping artefact similar to beam hardening in conventional x-ray CT (figure 2). When dense objects are inserted into an otherwise water-like phantom, the degree to which the relative scattering power underestimates the material specific ratio $X_{0, w} / X_{0}$ of radiation lengths depends on the depth of the inserts (figure 3).

In Monte Carlo simulated images of the Gammex phantom, the reconstructed relative scattering power in dense inserts was systematically lower than the characteristic radiation length ratio $X_{0, w} / X_{0}$ and systematically higher in adipose, breast, and brain inserts (figure 7), consistent with our theoretical analysis. The image of the spiral phantom showed that the reconstructed relative scattering power underestimates the ratio $X_{0, w} / X_{0}$ (4.06 for aluminium) even more so the deeper the inserts lie in the phantom. At the centre, the reconstructed value was about $20 \%$ lower than the theoretical $X_{0, w} / X_{0}$ (see upper right panel in figure 8).

One way to correct for the under estimation in dense material would be to exploit its similarity to beam hardening and adapt strategies typically used in x-ray CT. Another option is to rely on iterative reconstruction and explicitly model the energy-dependence of MCS based on some a priori knowledge, as proposed by Bopp et al. (2015). However, the MLP would need to be incorporated into the forward projection matrix to achieve best possible spatial resolution, which is probably more complicated in scattering proton $\mathrm{CT}$ than in energy-loss proton $\mathrm{CT}$ because the angular variance needs to be estimated from several trajectories at some point during the reconstruction.

We investigated the statistical properties of scattering proton CT and derived an expression for the intrinsic relative scattering power precision (section 2.5). At equal dose, the noise in the reconstructed scattering proton CT images was about 50 times higher than in the energy-loss image, but about 10 times lower than in the nuclear attenuation image. As a consequence, the SNR and CNR was 40-50 times lower in the reconstructed scattering image of the Gammex phantom than in the energy-loss image. The interesting conclusion here is: scattering proton CT promises to yield higher contrast at first sight because the ratio $X_{0, w} / X_{0}$ is larger for some dense materials than their RSP. This has been pointed out e.g. by Plautz et al. (2014). The true limiting factor in scattering proton CT, however, is the higher image noise so that SNR and CNR turn out to be less favourable. The practical advantage of a scattering proton CT scanner is that it does not require any device to measure proton residual energy, such as a calorimeter or range telescope. This might be reason enough in some circumstances to accept the disadvantages compared to energy-loss proton CT.

Helium or even heavier ions have been investigated as alternative particles for ion imaging (Parodi, 2014), with the former hypothesised to be optimal in terms of spatial resolution of the images (Collins-Fekete et al., 2016; Piersimoni et al., 2018). Studying these particles in the context of scattering proton CT in detail was beyond the scope of our work, but we share a few considerations here. The reason for potentially better spatial resolution in heavier ion CT is that MLP estimation is less uncertain for the heavier ions than for protons because they suffer less from MCS. If this is true for energy-loss CT, it will also be valid for scattering CT because the distance driven binning method is the same in both cases. Regarding noise properties for helium ions as an example, we refer to equation 26 and note that the prefactor containing the ratio of variance and scattering power is about $30 \%$ smaller for helium ions of initially $800 \mathrm{MeV}$ than for protons of initially $200 \mathrm{MeV}$ (equal range) behind a $20 \mathrm{~cm}$ water cylinder. At the same 
time, the stopping power at the cylinder centre (second factor in equation 26) is about a factor 4 larger for helium. The dose contribution due to nuclear interactions would require more detailed considerations, but will not change the order of magnitude of the overall expression. Therefore, we do not expect helium scattering $\mathrm{CT}$ to perform significantly better or worse than proton scattering $\mathrm{CT}$ in terms of image noise. It is worth mentioning in this context that electrons have also been explored for scattering CT imaging (Jansen et al., 2018), but due to their much different mass and energy (order of $\mathrm{GeV}$ ) we cannot immediately transfer our conclusions to that case.

A limitation of our Monte Carlo simulations is that we applied perfect particle filtering by removing all protons which underwent nuclear interactions in the phantom. In practice, such events can only be filtered out to some extent, typically by applying cuts on the angular and energy distributions (Schulte et al., 2008). As nuclear scattering is associated with large angle scattering, it is likely that this will lead to some sort of systematic misestimation of the angular variance and thus of the reconstructed relative scattering power. Investigating these aspects was beyond the scope of this work.

Furthermore, our analysis of image noise concentrated on the intrinsic statistical limitations of the modality and disregarded experimental uncertainties, in particular the tracking devices. Their impact can be estimated as follows: According to equation 26, image noise is approximately (within the Gaussian approximation of MCS) proportional to the variance of the deviation angles $\Delta \theta$. Compared to an ideal simulation, uncertainties in the measured entrance and exit angle widen the distribution of deviation angles $\Delta \theta$ and increase the effectively measured variance by $\sigma_{\text {exp }}^{2} \equiv \sigma_{\text {entry }}^{2}+\sigma_{\text {exit }}^{2}$, where $\sigma_{\text {entry }}$ and $\sigma_{\text {exit }}$ are the standard errors which quantify the tracker uncertainty. Image noise will increase proportionally. Measurement uncertainties will be important mainly in thin objects where the intrinsic angular variance due to MCS is small. The expected uncertainty of the angle measurement can be estimated based on the tracker geometry, material and the proton energy (Krah et al., 2018a). Using the Phase-II proton CT scanner prototype reported in Sadrozinski et al. (2013) as example, we calculated that noise in the scattering image of a water cylinder recorded at $200 \mathrm{MeV}$ would increase by $20 \%$ for a cylinder diameter of $2 \mathrm{~cm}$, but by less than $3 \%$ for a diameter of $10 \mathrm{~cm}$ or more. It is likely that heterogeneous phantom geometry and composition will contribute to image noise, as has been shown for energy-loss proton CT (Dickmann et al., 2019). The initial beam divergence does not impact image noise because the reconstruction uses the differences between exit and entry angles. Overall, the intrinsic image noise estimated in this work should be reasonably representative of the noise in a real scattering proton CT image. In the energy-loss proton CT image of a $10-20 \mathrm{~cm}$ thick phantom, on the other hand, contributions to image noise due to the beam's initial energy spread and the detection process are together of a similar magnitude as the intrinsic noise due to energy straggling considered in this work (Dickmann et al., 2019).

The main reason for the development of energy-loss proton CT is that it can directly provide an RSP map of a patient needed by proton therapy treatment planning systems (TPS). Scattering proton $\mathrm{CT}$ as described in this work does not provide this quantity, but rather a map of the material's (relative) radiation length $\left(X_{0, w} / X_{0}\right)$. At the same time, Monte Carlo TPS need more information about the tissue properties as input to the underlying physics models than RSP, e.g. elemental composition in Geant4 (Agostinelli et al., 2003) or directly radiation length in Fred (Schiavi et al., 2017). These additional quantities can be obtained through tabulated conversion from another quantity (Kanematsu et al., 2012). Alternatively, scattering proton CT could be combined with energy-loss proton CT, or even with x-ray based CT (Vilches-Freixas et al., 2018), to provide complementary information to the TPS. The accuracy of such a combined direct measurement would have to be at least as good as the one associated with a modelbased conversion. Investigating implementation details and whether the inferior image quality in scattering proton $\mathrm{CT}$ precludes its use in a clinical context was not the scope of this work.

We finally comment on the operating mode of the proton CT scanner: For both contrast 
mechanisms, operating a proton CT scanner in list-mode is demanding because the detection hardware needs to be extremely fast to keep up with the particle fluence of a therapeutic accelerator, even if operated at very low intensity (Johnson, 2018). As a technically simpler alternative, "integrated-mode" approaches have been studied for energy-loss proton CT and among them those where the imaged object is irradiated with pencil beams (Rescigno et al., 2015; Farace et al., 2016; Bentefour et al., 2016; Krah et al., 2018b) and the detector records the mean energy loss or residual range per pencil beam. In this sense, the averaging operation occurs physically as part of the detection process while it is done numerically during distance driven binning in list-mode operation. Indeed, Meyer et al. (2017) found comparable RSP accuracy with both operation modes for carbon ion CT, but we would expect a similar assessment for protons. Integrated mode scattering proton $\mathrm{CT}$ would require a detection device capable of measuring the angular variance (or another suitable quantity) per pencil beam directly instead of estimating it numerically during distance driven binning in list-mode. Whether new detector technology, e.g. high density scintillating glass (Wilkinson et al., 2017), might be suitable for this purpose and what accuracy is to be expected would require a dedicated study. Regardless of the contrast mechanism, integrated-mode scanners provide lower spatial resolution than list-mode scanners because reconstruction can only be performed along the average MLP of all particles in one beam and not each proton's MLP individually (Krah et al., 2018a).

\section{Conclusions}

In this work, we investigated a contrast mechanism of proton CT imaging which exploits the effect of multiple Coulomb scattering of protons in the imaged object. We showed that analytical reconstruction is possible by introducing a relative scattering power and developed a distancedriven filtered backprojection reconstruction, based on an algorithm previously proposed for energy-loss proton $\mathrm{CT}$, which makes use of the most likely path concept. We found that the properties of the relative scattering power can lead to an underestimation of relative scattering power in image regions denser than water and to artefacts which are somewhat similar to beam hardening in x-ray CT imaging. We showed that spatial resolution in scattering proton $\mathrm{CT}$ was similar to energy-loss proton CT. Intrinsic noise was observed to be substantially higher than in energy-loss proton CT (by a factor of 40-50 at the centre of a $20 \mathrm{~cm}$ water cylinder). Our analysis revealed that signal-to-noise and contrast-to-noise is likewise worse than in energy-loss by at least a factor of 40 despite the higher contrast in dense tissues, mainly because of the higher noise. The practical advantage of scattering over energy-loss proton CT is that it only needs tracker devices, but no energy sensitive detectors such as calorimeters. It could therefore be interesting as a complementary modality in certain settings despite its inferior performance.

\section{Acknowledgements}

The work of Nils Krah was partially supported by the project DIC20161236452 of the Fondation pour la Recherche Médicale and partially by funding from the European Union's Horizon 2020 research and innovation programme under the Marie Sklodowska-Curie grant agreement No 753370. This work was performed within the framework of the LABEX PRIMES (ANR11-LABX-0063) of Université de Lyon, within the program "Investissements d'Avenir" (ANR-11IDEX-0007) operated by the French National Research Agency (ANR). We also gratefully acknowledge the support from the CNRS/IN2P3 Computing Center (Lyon/Villeurbanne - France), for providing a significant amount of the computing resources needed for this work. 


\section{References}

Agostinelli, S. et al. (2003). "GEANT4 - A simulation toolkit". In: Nuclear Instruments and Methods in Physics Research, Section A: Accelerators, Spectrometers, Detectors and Associated Equipment 506.3, pp. 250-303. ISSN: 01689002. DOI: 10.1016/S0168-9002(03)01368-8.

Arbor, N. et al. (2015). "Monte Carlo comparison of x-ray and proton CT for range calculations of proton therapy beams". In: Physics in Medicine and Biology 60.19, p. 7585. ISSN: 0031-9155. DOI: $10.1088 / 0031-9155 / 60 / 19 / 7585$.

Bentefour, E. H. et al. (2016). "Concept of proton radiography using energy resolved dose measurement". In: Physics in Medicine and Biology 61.16, N386-N393. ISSN: 13616560. DOI: 10.1088/0031-9155/61/16/N386.

Berger, M. et al. (2005). ESTAR, PSTAR, and ASTAR: Computer Programs for Calculating Stopping-Power and Range Tables for Electrons, Protons, and Helium Ions (version 1.2.3). DOI: $10.18434 / \mathrm{T} 4 \mathrm{NC} 7 \mathrm{P}$.

Bopp, C et al. (2013). "Proton computed tomography from multiple physics processes". In: Physics in Medicine and Biology 58.20, pp. 7261-7276. ISSN: 0031-9155. DOI: 10.1088/00319155/58/20/7261.

Bopp, C et al. (2015). "Quantitative proton imaging from multiple physics processes: a proof of concept." In: Physics in medicine and biology 60.13, pp. 5325-41. ISSN: 1361-6560. DOI: $10.1088 / 0031-9155 / 60 / 13 / 5325$.

Collins-Fekete, C.-A. et al. (2016). "A theoretical framework to predict the most likely ion path in particle imaging". In: Physics in Medicine \& Biology 62.October, pp. 1777-1790.

Collins-Fekete, C.-A. et al. (2017). "Pre-treatment patient-specific stopping power by combining list-mode proton radiography and x-ray CT". In: Physics in Medicine 85 Biology 62.17, pp. 6836-6852. ISSN: 1361-6560. DOI: 10.1088/1361-6560/aa7c42.

Cormack, A. M. (1963). "Representation of a Function by Its Line Integrals, with Some Radiological Applications". In: Journal of Applied Physics 34.9, pp. 2722-2727. ISSN: 0021-8979. DOI: $10.1063 / 1.1729798$.

Cormack, A. M. et al. (1976). "Quantitative proton tomography: preliminary experiments". In: Physics in Medicine and Biology 21.4, p. 560.

Dickmann, J. et al. (2019). "Prediction of image noise contributions in proton computed tomography and comparison to measurements". In: Physics in Medicine ES Biology 64.14, p. 145016. ISSN: 1361-6560. DOI: 10.1088/1361-6560/ab2474.

Erdelyi, B. (2009). "A comprehensive study of the most likely path formalism for protoncomputed tomography". In: Phys. Med. Biol. Phys. Med. Biol 54.54, pp. 6095-6095. Issn: 0031-9155. DOI: 10.1088/0031-9155/54/20/005.

Farace, P. et al. (2016). "Pencil beam proton radiography using a multilayer ionization chamber". In: Physics in medicine and biology 61.11, pp. 4078-87. ISSN: 1361-6560. DOI: 10.1088/00319155/61/11/4078.

Feldkamp, L. a. et al. (1984). "Practical cone-beam algorithm". In: Journal of the Optical Society of America A 1.6, p. 612. ISSN: 1084-7529. DOI: 10.1364/JOSAA.1.000612.

Gore, J. C. et al. (1978). "Statistical limitations in computed tomography". In: Physics in Medicine and Biology 23.6, p. 014. ISSN: 00319155. DOI: 10.1088/0031-9155/23/6/014.

Gottschalk, B. et al. (1993). "Multiple Coulomb scattering of $160 \mathrm{MeV}$ protons". In: Nuclear Instruments and Methods in Physics Research Section B: Beam Interactions with Materials and Atoms 74.4, pp. 467-490. ISSN: 0168583X. DOI: 10.1016/0168-583X (93) 95944-Z.

Gottschalk, B. (2010). "On the scattering power of radiotherapy protons." In: Medical physics 37.1, pp. 352-367. ISSN: 00942405. DOI: 10.1118/1.3264177.

Hanson, K. M. et al. (1981). "Computed tomography using proton energy loss". In: Phys. Med. Biol. 26, pp. 965-983.

Hanson, K. M. et al. (1982). "Proton computed tomography of human specimens". In: Phys. Med. Biol. 27, pp. 25-36. 
Highland, V. L. (1975). "Some practical remarks on multiple scattering". In: Nuclear Instruments and Methods 129.2, pp. 497-499.

Jansen, H. et al. (2018). "Feasibility of track-based multiple scattering tomography". In: Applied Physics Letters 112.14, p. 144101. ISSN: 0003-6951. DOI: 10.1063/1.5005503.

Johnson, R. P. (2018). "Review of medical radiography and tomography with proton beams". In: Reports on Progress in Physics 81.1, p. 016701. ISSN: 0034-4885. DOI: 10.1088/1361$6633 / \mathrm{aa} 8 \mathrm{~b} 1 \mathrm{~d}$.

Kanematsu, N. et al. (2012). "Relationship between electron density and effective densities of body tissues for stopping, scattering, and nuclear interactions of proton and ion beams". In: Medical Physics 39.2, pp. 1016-1020. ISSN: 00942405. DOI: 10.1118/1.3679339.

Khellaf, F. et al. (2020). "2D directional ramp filter". In: Physics in Medicine $\mathscr{6}$ Biology 65.8, 08NT01. ISSN: 1361-6560. DOI: 10.1088/1361-6560/ab7875.

Koehler, A. M. (1968). "Proton Radiography". In: Science 160.3825, pp. 303-304. ISSN: 0036-8075. DOI: $10.1126 /$ science.160.3825.303.

Krah, $\mathrm{N}$ et al. (2018a). "A comprehensive theoretical comparison of proton imaging set-ups in terms of spatial resolution". In: Physics in Medicine $\&$ Biology 63.13, p. 135013. ISSN: 13616560. DOI: $10.1088 / 1361-6560 /$ aaca1f.

Krah, N. et al. (2018b). "Proton radiography with a commercial range telescope detector using dedicated post processing methods". In: Physics in Medicine $E$ B Biology 63.20, p. 205016. ISSN: 1361-6560. DOI: 10.1088/1361-6560/aae043.

Krah, N. et al. (2019). "Polynomial modelling of proton trajectories in homogeneous media for fast most likely path estimation and trajectory simulation". In: Physics in Medicine 83 Biology 64.19, p. 195014. ISSN: 1361-6560. DOI: 10.1088/1361-6560/ab3d0b.

Li, T. et al. (2006). "Reconstruction for proton computed tomography by tracing proton trajectories: A Monte Carlo study". In: Medical Physics 33.3, pp. 699-706. ISSN: 00942405. DOI: 10.1118/1.2171507.

Malmer, C. J. (2001). "ICRU Report 63. Nuclear Data for Neutron and Proton Radiotherapy and for Radiation Protection". In: Medical Physics 28.5, pp. 861-861. ISSN: 00942405. DOI: 10.1118/1.1369116.

Meyer, S. et al. (2017). "Comparative Monte Carlo study on the performance of integrationand list-mode detector configurations for carbon ion computed tomography". In: Physics in Medicine ES Biology 62.3, p. 1096.

Paganetti, H. (2012). Proton Therapy Physics. Ed. by H. Paganetti. Boca Raton: Taylor \& Francis Group. ISBN: 9781439836453.

Parodi, K. (2014). "Heavy ion radiography and tomography". In: Physica Medica 30.5, pp. 539543. ISSN: 1724191X. DOI: 10.1016/j.ejmp.2014.02.004.

Piersimoni, P. et al. (2018). "Helium CT: Monte Carlo simulation results for an ideal source and detector with comparison to proton CT"'. In: Medical Physics 45.7, pp. 3264-3274. ISSN: 0094-2405. DOI: $10.1002 / \mathrm{mp} .12942$.

Plautz, T. et al. (2014). "200 MeV Proton Radiography Studies With a Hand Phantom Using a Prototype Proton CT Scanner". In: IEEE Transactions on Medical Imaging 33.4, pp. 875881. ISSN: 0278-0062. DOI: 10.1109/TMI .2013.2297278.

Poludniowski, G et al. (2014). "Proton computed tomography reconstruction using a backprojectionthen-filtering approach". In: Physics in Medicine 83 Biology 59.24, p. 7905.

Poludniowski, G. et al. (2015). "Proton radiography and tomography with application to proton therapy". In: The British Journal of Radiology 88.1053, p. 20150134. ISSN: 0007-1285. DOI: 10.1259/bjr. 20150134.

Quiñones, C. T. et al. (2016). "Filtered back-projection reconstruction for attenuation proton CT along most likely paths". In: Physics in Medicine and Biology 61.9, pp. 3258-3278. ISSN: 0031-9155. DOI: 10.1088/0031-9155/61/9/3258. 
Rädler, M. et al. (2018). "Two-dimensional noise reconstruction in proton computed tomography using distance-driven filtered back-projection of simulated projections". In: Physics in Medicine \& Biology 63.21, p. 215009. ISSN: 1361-6560. DOI: 10.1088/1361-6560/aae5c9.

Rao, C. R., ed. (1973). Linear Statistical Inference and its Applications. Wiley Series in Probability and Statistics. Hoboken, NJ, USA: John Wiley \& Sons, Inc. ISBN: 9780470316436. DOI: 10.1002/9780470316436.

Rescigno, R. et al. (2015). "A pencil beam approach to proton computed tomography." In: Medical physics 42.11, pp. 6610-24. ISSN: 0094-2405. DOI: 10.1118/1.4933422.

Richard, S. et al. (2012). "Towards task-based assessment of CT performance: System and object MTF across different reconstruction algorithms". In: Medical Physics 39.7, pp. 4115-4122. ISSN: 00942405. DOI: 10.1118/1.4725171.

Rit, S. et al. (2013). "Filtered backprojection proton CT reconstruction along most likely paths." In: Medical physics 40.3, p. 031103. ISSN: 0094-2405. DOI: 10.1118/1.4789589.

Sadrozinski, H. F.-W. et al. (2013). "Development of a Head Scanner for Proton CT". In: Nuclear instruments 83 methods in physics research. Section A, Accelerators, spectrometers, detectors and associated equipment 699, pp. 205-210. ISSN: 0168-9002. DOI: 10.1016/j.nima. 2012. 04.029.

Sarrut, D. et al. (2014). "A review of the use and potential of the GATE Monte Carlo simulation code for radiation therapy and dosimetry applications". In: Medical Physics 41.6Part1, p. 064301. ISSN: 00942405. DOI: 10.1118/1.4871617.

Schiavi, A. et al. (2017). "Fred: A GPU-accelerated fast-Monte Carlo code for rapid treatment plan recalculation in ion beam therapy". In: Physics in Medicine and Biology 62.18, pp. 7482 7504. ISSN: 13616560. DOI: 10.1088/1361-6560/aa8134.

Schulte, R. W. et al. (2008). "A maximum likelihood proton path formalism for application in proton computed tomography". In: Medical Physics 35.11, p. 4849. ISSN: 00942405. DOI: $10.1118 / 1.2986139$.

Schulte, R. W. et al. (2005). "Density resolution of proton computed tomography". In: Medical Physics 32.4, pp. 1035-1046. ISSN: 00942405. DOI: 10.1118/1.1884906.

Schulte, R. W. et al. (2012). "Proton CT for Improved Stopping Power Determination in Proton Therapy, invited." In: Transactions of the American Nuclear Society 106, pp. 55-58. ISSN: 0003-018X.

Taylor, J. T. et al. (2016). "An experimental demonstration of a new type of proton computed tomography using a novel silicon tracking detector". In: Medical Physics 43.11, pp. 6129-6136. ISSN: 00942405. DOI: 10.1118/1.4965809.

Vilches-Freixas, G. et al. (2018). "Deriving the mean excitation energy map from dual-energy and proton computed tomography". In: Physics and Imaging in Radiation Oncology 6, pp. 20-24. ISSN: 24056316. DOI: $10.1016 / j \cdot$ phro.2018.04.001.

Wilkinson, C. J. et al. (2017). "High density scintillating glass proton imaging detector". In: ed. by T. G. Flohr et al., p. 101323V. DOI: 10.1117/12.2252777.

Williams, D. C. (2004). "The most likely path of an energetic charged particle through a uniform medium". In: Physics in Medicine and Biology 49.13, pp. 2899-2911. ISSN: 0031-9155. DOI: 10.1088/0031-9155/49/13/010. 